\title{
An active UHF RFID localization system for fawn saving
}

\author{
M. Eberhardt, M. Lehner, A. Ascher, M. Allwang, and E. M. Biebl \\ Fachgebiet Höchstfrequenztechnik, Technische Universität München, Munich, Germany \\ Correspondence to: M. Eberhardt (michael.eberhardt@ tum.de)
}

Received: 28 November 2014 - Revised: 23 February 2015 - Accepted: 6 March 2015 - Published: 3 November 2015

\begin{abstract}
We present a localization concept for active UHF RFID transponders which enables mowing machine drivers to detect and localize marked fawns. The whole system design and experimental results with transponders located near the ground in random orientations in a meadow area are shown. The communication flow between reader and transponders is realized as a dynamic master-slave concept. Multiple marked fawns will be localized by processing detected transponders sequentially. With an eight-channelreceiver with integrated calibration method one can estimate the direction-of-arrival by measuring the phases of the transponder signals up to a range of $50 \mathrm{~m}$ in all directions. For further troubleshooting array manifolds have been measured. An additional hand-held receiver with a two-channel receiver allows a guided approaching search without endangering the fawn by the mowing machine.
\end{abstract}

\section{Introduction}

In the spring, when the vegetation increases, the high season of forage crop for farmers begins. The time pressure is high and the technology enables very large mowing performance. At the same time it is the main period for fawns to be born (Rieck, 1955). The does look for calm and safe places and prefer meadows nearby a border of the wood. The high grass affords an excellent protection against natural threats. In addition, the fawns have a native instinct in the first weeks of their lives (Jarnemo, 2002). They press down flat on the ground if any danger is approaching and keep calm even when a mowing-machine runs over them. Many fawns are killed every year in this way (Jarnemo, 2002).

In Germany, the farmers are obliged by law to avoid injuring animals. To date, the fields will be searched on foot for fawns, which means a considerable expense. Many researches have been made for a fawn detection technology.
Developed systems in the recent years based on infrared are shown in Tank et al. (1992) and Israel (2011) or based on radar in Fackelmeier and Biebl (2009) and Reichthalhammer (2012). These technologies facilitate the search substantially. Nevertheless the search performance is still insufficient compared to peak mowing performance caused by many farmers mowing at the same time. Another drawback is possible false-positive alarms, which are very user-unfriendly because they slow the searching process in addition. Thus, there is a need for a system which allows to separate the search for fawns from the mowing.

To overcome this problem, a new approach is a fourstep procedure to separate the searching- from the mowingprocess. The four steps are Finding, Marking, Recovering and Saving. In this way any searching technology, whether based on infrared or radar, can be used to find the fawns several days before the mowing. After finding the fawns the first time, one can mark them with a RFID-transponder. Marked fawns can be reliably detected during the mowing without false-positive alarms and localized to secure them out of the danger area. This approach expands the existing searching technologies to a very flexible fawn saving concept.

In this paper we present a first RFID system prototype for the mentioned fawn saving concept and a direction finding system to localize the RFID transponders. The direction finding system consists of a vehicle mounted and a handheld receiver for estimating the direction-of-arrival of active RFID transponders in the UHF band. The mowing machine mounted system measurements have been performed under real world conditions, in which transponders were located near the ground, in random orientations and short distances up to $50 \mathrm{~m}$. The hand-held receiver has been tested under simple conditions. 


\section{Preliminary}

The intent is to develop a RFID localization system which is able to gather information about the whole working area around the mowing machine. A mowing machine driver shall be aware if there is a marked fawn and if there is one, where it is located. Transponders, which will be detected beside the mowing-machine's current lane, could be registered for the next turn and used as backup information.

To simplify the use case and the measurements, we assumed a mowing machine not moving, in a wide, open and flat meadow area surrounded by a couple of fawns on random positions and random orientations. If a doe bears more than one fawn, than they can lay very close to each other.

In practice, there is only one useful position on mowing machines for a sensor node; the vehicle's roof. With one sensor node the only way to determine a relative position of a transponder is estimating the distance and the direction-ofarrival (DOA). In this paper the focus is set only on DOA and a two step procedure is presented to find a transponder only by bearing information under assistance of a hand-held receiver. Due to the possible short distance between several fawns, a classical DOA device would need a very high resolution.

A detected transponder can be localized in two steps only by getting bearing information. First step is a first estimation of the DOA by the sensor node of the mowing machine. With a known direction to a marked fawn, the driver can stop the machine and start the second step, an approach to the fawn guided through hand-held receiver. In waist-high grass a person can not hold a precise walking direction over a distance longer than $10 \mathrm{~m}$. This reveals the need for a device that corrects in real-time the walking direction. The corrected bearing information given by the hand-held receiver enables a person to approach up to a needed distance of $0.5 \mathrm{~m}$.

The increasing speed of mowing-machines up to $20 \mathrm{~km} \mathrm{~h}^{-1}$ and a receiving antenna fixed in the center of the machine lead to a minimum range of at least $15 \mathrm{~m}$ in driving direction. Today's deckle width are up to $9 \mathrm{~m}$. The transmit power regulations for RFID applications are limited to $2 \mathrm{~W}$ ERP in many countries. Therefore, a passive UHF RFIDtransponder with a matched dipole antenna reaches a maximum read range of $11 \mathrm{~m}$ under good conditions (Finkenzeller, 2008). Due to a given antenna mismatch caused by the fawn body, the position near above the ground and random orientation of dipole antenna, a passive RFID system can be excluded. By using an active RFID transponder, the possible range for a certain detection is expected to be much larger and the antenna mismatch on the transponder side is a second-tier problem.

An active RFID transponder requires a special communication concept. The transponder's maximum lifetime is limited on its battery capacity. A needed lifetime of at least eight weeks will not be realizable in a small transponder with small battery, which is in receive mode for eight weeks. Therefore the transponder has to be in a sleep mode, wakes up frequently, sends a short beacon, waits a short duration for a response and goes back to sleep mode. With a limited capacity the lifetime depends mainly on beacon-sending-interval and receiving-duration. On the other hand, the detection duration depends on the sending-interval, too.

\section{Communication concept}

The bottleneck is the very small transponder size, which has to be able to be clipped to a fawn. In future, it is planned to design small transponders with integrated communication ICs, small chip antennas and small batteries. Almost all of the time the reader interface on the mowing machine will be out of range of the marked fawns. Thus, the transponder has to be nearly all of its lifetime in a sleep mode to save enough power for a maximum service life (power mode 2, PM2). If a transponder and the reader are in range, they have to detect each other as fast as possible because of the fast mowing speed.

For this reason the communication concept was designed based on two aspects; the maximum possible lifetime and the maximum response duration, which is needed for detecting the transponder. A long transponder lifetime due to limited battery capacity is important because of the varying birth period of the fawns and the weather which changes the mowing period. The maximum duration for detecting the transponder depends on the mowing speed and the range of the RFIDsystem. With a safety distance of $20 \mathrm{~m}$, a range of $50 \mathrm{~m}$, a braking distance of $10 \mathrm{~m}$ and the moving speed of $20 \mathrm{~km} \mathrm{~h}^{-1}$, it leads to a maximum detection duration of $3.6 \mathrm{~s}$.

Figure 1 shows the first approach for the transponder behavior. After assembly, the transponder is in the "Initialization State" which is a deep sleep mode (power mode 3, PM3) with lowest possible current consumption until it is clipped on a fawn and it is initialized for usage. This can not be done by a timer but only by an external action like a button or a magnetic switch. From this point, the transponder is in the "Basic State" and it reacts as a master and will frequently sent a beacon with its ID. It is not possible to fall back to "Initialization State". If the reader is not in range, which is true for most of its lifetime, then the transponder will fall back to a regular sleep mode (PM2) to save battery power. If the reader gets in range, it can react and set the transponder by command in the "Second State". Now the transponder is the slave and the reader is the master for this registered transponder.

By doing this, possible collisions between several transponders can be avoided and all further communications and interactions will be controlled by the reader. Multiple registered transponders can be sequentially processed and to each the DOA determined. Later, the results will show that every DOA-algorithm is working well in this scenario. By determining the DOA to each transponder sequentially 


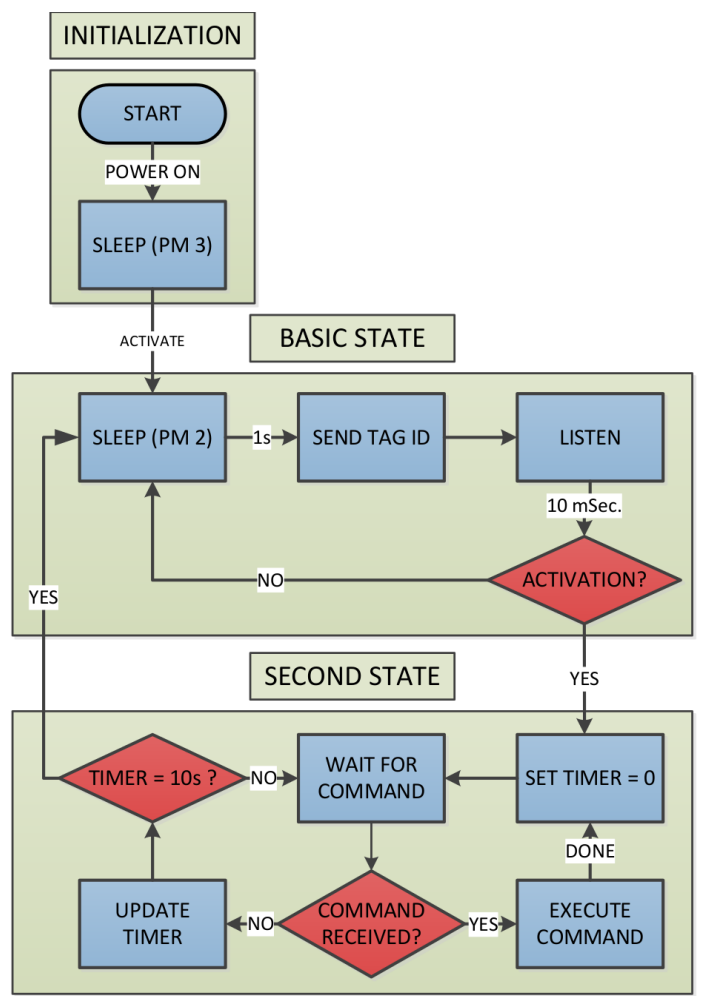

Figure 1. Transponder firmware states and work flow.

the limited resolution of simple algorithms like beamforming and correlative interferometery is sufficient and computation power can be saved.

At the moment, there is no further state for transponder recycling. But it is conceivable to implement a further process for detecting low battery capacity, which sets the transponder in a "Recycle State". In the "Recycle State", the beacon interval could be much longer. This function may be needed to be able to find the transponders, which are no longer attached to a fawn. Due to missing experience a standard return procedure for all used transponders is not projectable at the moment.

\section{Theory of operation for DOA estimation}

There are a lot of different algorithms to estimate the DOA of an impinging electromagnetic wave. Zekavat and Buehrer (2011) splits them into delay-and-sum algorithms and those with high resolution like MUSIC and ESPRIT. All of them need an array manifold which is defined as

$\mathbf{A}=\left[\boldsymbol{a}\left(\theta_{1}\right) \boldsymbol{a}\left(\theta_{2}\right) \ldots \boldsymbol{a}\left(\theta_{n}\right)\right]$, where

$\boldsymbol{a}\left(\theta_{n}\right)=\left[\begin{array}{c}1 \\ e^{j \Delta \Phi_{2}} \\ e^{j \Delta \Phi_{3}} \\ \ldots \\ e^{j \Delta \Phi_{M}}\end{array}\right]$

is the direction-dependent steering vector and $\theta$ is the azimuthal DOA. The terms $\Delta \Phi_{n}$ are the phase-differences between the antenna $n$ and the first antenna element in an array with $M$ elements. How many columns (steering-vectors) in the manifold are needed depends on the used algorithm.

Here we choose for first experimental results some known algorithms, for example the Bartlett-Beamformer, which is defined as

$\mathbf{P}(\theta)=\boldsymbol{a}(\theta)^{\mathrm{H}} \cdot \mathbf{R}_{\mathrm{XX}} \cdot \boldsymbol{a}(\theta)$,

where $\mathbf{R}_{\mathrm{XX}}$ is the signal covariance matrix of the $M$ received signals. A more simpler way is to correlate all steering vectors with one measured steering vector. The phasedifferences can be extracted in the first column of the signal covariance matrix or from the complex spectrum of each channel.

$\mathbf{P}=\left(\boldsymbol{a}_{\text {measured }}^{\mathrm{H}} \cdot \mathbf{A}\right)^{\mathrm{T}}$

This method is sometimes called correlative interferometery and is very similar to the Bartlett-Beamformer. The well known MUSIC algorithm with high resolution is defined as

$\mathbf{P}(\theta)=\frac{1}{\boldsymbol{a}(\theta)^{\mathrm{H}} \mathbf{E}_{\mathrm{N}} \mathbf{E}_{\mathrm{N}}^{\mathrm{H}} \boldsymbol{a}(\theta)}$,

where $\mathbf{E}_{\mathrm{N}}$ is a matrix with noise eigenvectors (Schmidt, 1986). All algorithms have in common to take samples, calculating the signal covariance matrix and estimating angledependent power with the array manifold.

Typical error sources are disrupted wave propagation, unknown parameters about the target signal, co-channel interference, noise caused by the receiver, measurement errors and modeling errors (Tuncer and Friedlander, 2009). The wave propagation conditions in the open meadow area without obstacles are very good, so only influences from the ground and possible diffractions by the mowing-machine are expected. The transponders work cooperative, so all parameters about the signals are known. Measurement errors can be avoided very well by calibrating the receiver with a reference signal. The architecture, as shown in Fig. 2, is realized with high frequency switches to select either the signal received by the antennas or a reference signal generated by a third local oscillator. The main problem is the modeling errors due the antenna array.

Typical modeling errors are due to mutual coupling between antenna elements, coupling between antenna elements 


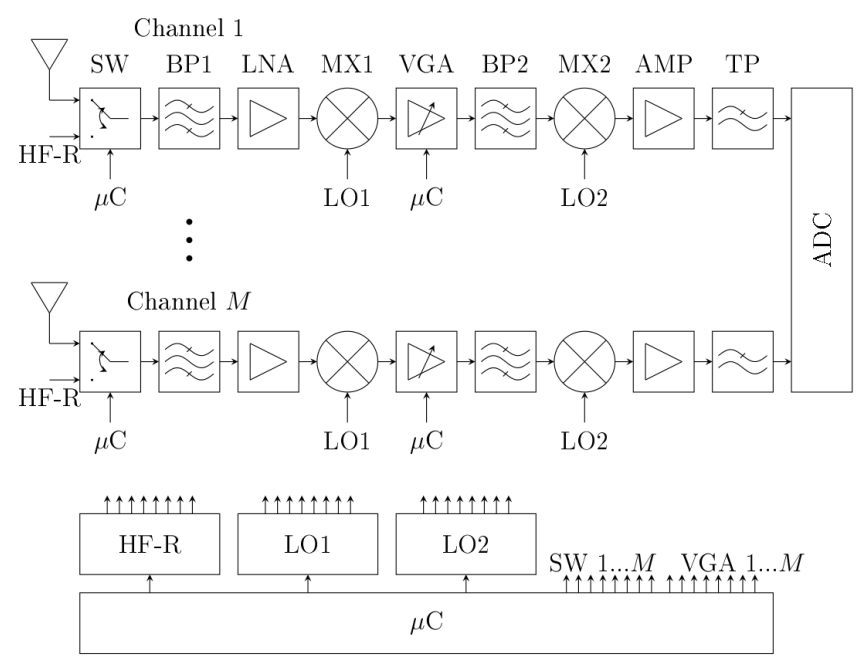

Figure 2. Receiver architecture schema of 8 channel receiver and 2 channel receiver with integrated calibration device.

and mechanical support structures and reflection and diffraction caused by the objects near the antenna array (Tuncer and Friedlander, 2009). The mowing machines are up to $3.5 \mathrm{~m}$ high and the limit in Germany for vehicles is $4 \mathrm{~m}$. For this reason we tried to mount the antenna array very close to the vehicle's roof. The expectation is that this will lead to the biggest error source. Furthermore, there will be non-line-ofsight connections to some measurement points depending on transponder distance and roof size.

A possible approach to compensate the expected errors could be done by measuring many reference points with known directions (Pierre and Kaveh, 1995). For every reference point a carrier is sent by an oscillator connected to a dipole antenna and the DOA-system samples the received signals on each channel. Then we build for every reference direction a measured steering vector and build the array manifold.

For reference measurements it is important to know a minimum distance between receiving antennas from the direction-finding system and the source antenna. A minimum distance can be calculated by Tuncer and Friedlander (2009):

$d \geq \frac{\pi A^{2}}{4 \delta_{\mathrm{tol}} \lambda}$,

where $A$ is the aperture diameter, $\lambda$ the shortest operated wavelength and $\delta_{\text {tol }}$ the tolerable phase deviation at the antenna array's edge elements. With $\delta_{\text {tol }}=2.5^{\circ}$ and $A=0.5 \mathrm{~m}$ leads to $d \geq 12.99 \mathrm{~m}$.

\section{Hardware setup}

This section gives a short overview of all relevant hardware realizations. There are the RFID communication system, the eight-channel-receiver and a uniform circular antenna array

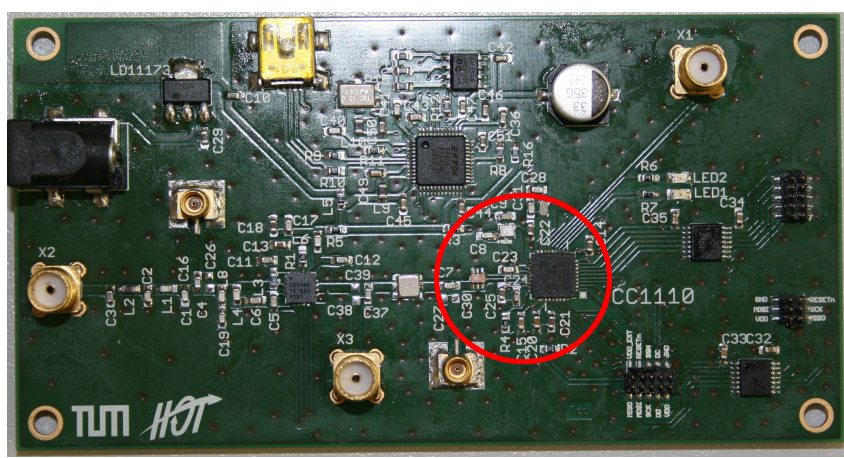

Figure 3. Used RFID-reader module with power amplifier and connectors. Red mark shows needed elements for a smaller transponder.

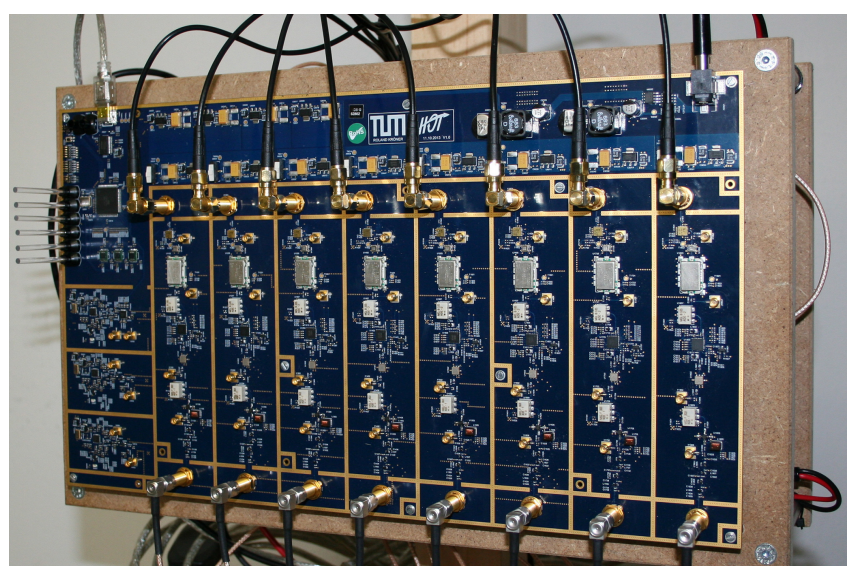

Figure 4. 8 channel receiver with integrated calibration device for mowing machine mounted DOA-system.

for the mowing machine mounted DOA system and a handheld receiver prototype for the guided approach.

For the communication we choose a fully integrated communication chip, the CC1110 from Texas Instruments, because of the very small current consumption and different programmable power modes. A very powerful reader device which in the beginning can be used as transponder as well, is shown in Fig. 3. In the red circle, the components are marked, which are only needed for a small transponder in the future. The additional components in this realization are a frontend amplifier and several connections like USB, UART and SPI. So, it is conceivable to be able to design a very small transponder, which can be clipped to a fawn's ear.

The detection range of the RFID-system was determined experimentally. A reader module with additional amplifier has been mounted on a mowing machines roof in $3 \mathrm{~m}$ height and a transponder without amplifier was placed right on the ground. The transponder's sending power was $10 \mathrm{dBm}$ and a certain detection range of at least $300 \mathrm{~m}$ could be reached. Via a SMA connector a trigger pulse for incoming transponder signals can be generated. The analog-digital-converters 


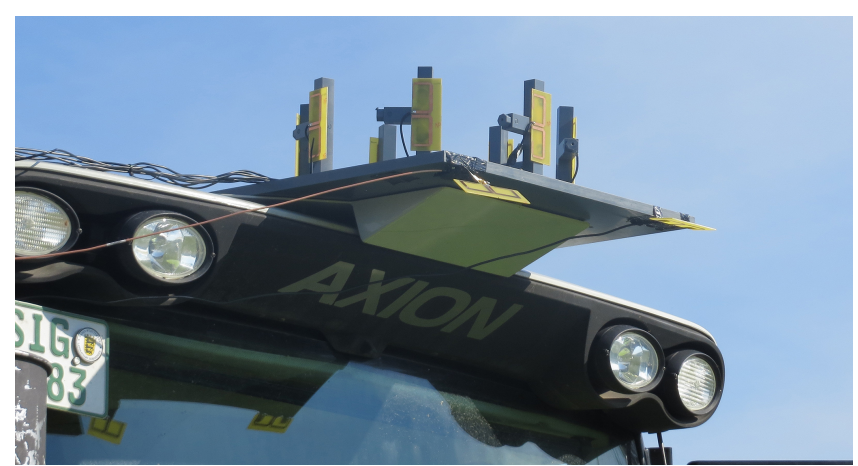

Figure 5. Uniform circular array prototype with 7 dipole elements, mounted on mowing machines roof. Two additional antennas for RFID-reader communication and received signal strength measurement.

(ADC) can trigger on this pulse and only incoming signals will be sampled and very efficient timings are possible.

The proposed eight-channel-receiver was constructed and is shown in Fig. 4. The hardware was optimized for receiving narrowband signals between 865 and $868 \mathrm{MHz}$ and convert it to an intermediate frequency between 1 and $8 \mathrm{MHz}$. Using a third local oscillator and a network with power dividers, a precise reference signal can be fed in over the switches to calibrate all channels and compensate the phase errors caused by the receiver hardware. Reference signals themselves have a maximum phase error of $1.5^{\circ}$, which was measured by a network analyzer. The known phase errors of the reference signals in each channel are factored in the whole phase error correction. A baseband conversion with IQ-modulator is not necessary. The complex analytical signal can be computed with the Hilbert transform and several ADCs for Q channels can be saved. The ADC is realized by a TI development kit with the AD5292 which was connected to a PC.

An antenna array prototype was realized without paying attention on high precise symmetry and is shown in Fig. 5. It is a uniform circular array with seven folded dipole elements. The apertures diameter is $40 \mathrm{~cm}$ which matches a relation of $\frac{d}{\lambda}=0.5$ between neighbor elements. The smallest distance between two elements in propagation direction matches $\frac{d}{\lambda}=$ 0.21 .

The hand-held receiver prototype was built up with a twochannel-receiver which has the same architecture shown in Fig. 2. The ADC was built with the AD9231 by Analog Devices and is realized as extension on an Arduino Due board. The hand-held receiver only needs to cover a sector in walking direction, so patch antennas by Kathrein as linear array with two elements are used. The whole prototype is shown in Fig. 6.

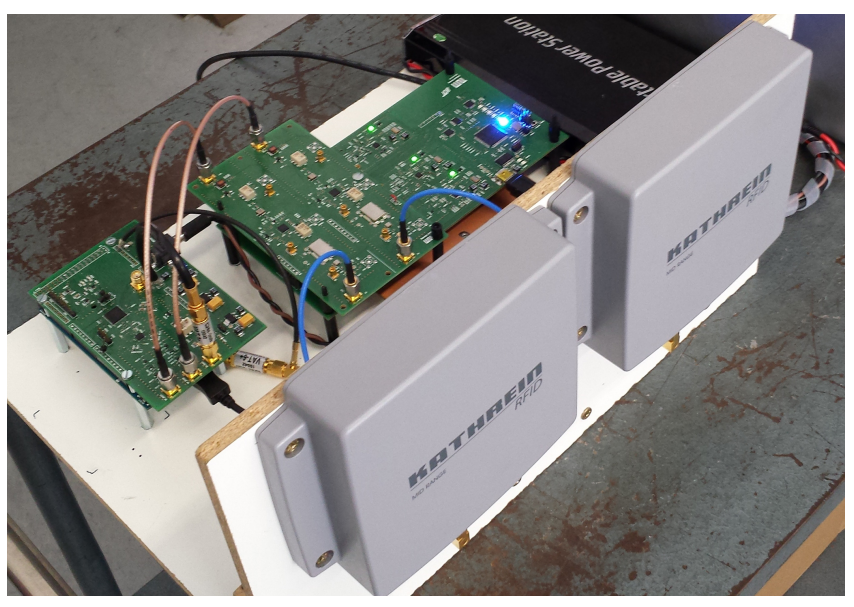

Figure 6. Prototype of hand-held receiver with 2 channel receiver and patch antenna array.

\section{Measurements and experimental results}

Experimental tests with the mowing machine mounted device have been made in an open meadow environment. The hand-held receiver was initially tested in an outdoor environment under simple conditions. The setups and results will be shown in this section.

\subsection{Mowing machine mounted device}

An initial stand-alone test with the eight-channel-receiver and the uniform circular array (UCA) was done in an outdoor environment. A transmitter oscillator with a dipole antenna and the UCA from the direction-finding system were arranged in $1 \mathrm{~m}$ height and $15 \mathrm{~m}$ distance. Within a maximum tolerance of $\pm 10^{\circ}$ every measurement was a correct DOA estimation with a mean error of $3^{\circ}$ without paying attention on high precise symmetry in the UCA and mutual coupling compensation between antenna elements.

For use case conditions, the presented antenna array was mounted close to a mowing machines roof. The total distance between a dipole element and the roof was $4 \mathrm{~cm}$, the vehicle roof height was $3.2 \mathrm{~m}$. For the calibration, we took a regular oscillator with the carrier frequency of $868 \mathrm{MHz}$ and a dipole antenna vertically orientated. It was mounted in $1 \mathrm{~m}$ height in $10^{\circ}$-steps and a distance of $15 \mathrm{~m}$ around the mowing machine. For every direction the signal was captured with 4096 samples and the steering vector calculated. Later the manifold was algorithm-dependent interpolated to be able to reach a theoretical accuracy of $0.1^{\circ}$.

In the test scenario a transponder was positioned in various directions with a horizontal distance to the antenna array of $15 \mathrm{~m}$ and $30 \mathrm{~m}$. The dipole antenna height above ground was $7 \mathrm{~cm}$ and aligned in various orientations on one measurement position. In some exemplary measurements, a maximum distance of $50 \mathrm{~m}$ was used. The detection of transponder by the 
Table 1. Correctly estimated positions with tolerance and used array-manifold, mowing machine mounted system.

\begin{tabular}{lrrr}
\hline & $p\left(5^{\circ}\right)[\%]$ & $p\left(10^{\circ}\right)[\%]$ & $p\left(15^{\circ}\right)[\%]$ \\
\hline Ideal manifold & 25.77 & 50.92 & 64.42 \\
Free manifold & 32.52 & 57.67 & 76.67 \\
Mounted manifold & 49.69 & 74.23 & 83.44 \\
\hline
\end{tabular}

Table 2. Mean error of correctly estimated positions and used arraymanifold, mowing machine mounted system.

\begin{tabular}{lrrr}
\hline & $p\left(5^{\circ}\right)\left[^{\circ}\right]$ & $p\left(10^{\circ}\right)\left[^{\circ}\right]$ & $p\left(15^{\circ}\right)\left[^{\circ}\right]$ \\
\hline Ideal manifold & 2.1 & 5.6 & 7.1 \\
Free manifold & 2.9 & 5.0 & 6.8 \\
Mounted manifold & 2.0 & 4.0 & 4.8 \\
\hline
\end{tabular}

reader worked without problems, so signals have been captured by the direction-finding system every time. Due to the short distances, the received power was high enough with signal-to-noise-ratios of at least $25 \mathrm{~dB}$. A total of 163 measurements were made. Each measurement was tested with every algorithm mentioned in Sect. 4 . The number of samples were varied via software between 2 and 1024 .

Before discussing the results, it is important to know that every algorithm estimated nearly the same result in every measurement position. In some cases a direction estimation difference of $0.5^{\circ}$ occurred, which is negligible. This shows that a simple algorithm is adequate for this problem and the liability to errors is due to a corrupted array manifold. Because of this, only results in dependency of the used manifold are presented. With at least 16 samples the results are identical. Now the captured samples are evaluated with different manifolds.

Table 1 shows the percentage of correctly estimated directions as a function of the tolerance. At first we tried to estimate the DOA with the ideal manifold for a UCA, which was just computed. For example, with a tolerance of $\pm 10^{\circ}$ $50.92 \%$ of the 163 measurements were correctly estimated. Table 2 shows the mean direction error additional for the correctly estimated positions.

Then two different calibrations are proceeded. The first one was a calibration, where the UCA was mounted on a tripod in an open meadow area. From this calibration we calculated a manifold, which is in Table 1 and 2 signed as "Free manifold". A second calibration was done with the UCA mounted close on the mowing machine's roof, which is signed as "Mounted manifold".

It is obvious that the ideal manifold provides the worst results and with the calibration with the mounted UCA the best. With the ideal manifold no mutual coupling or distortions by the vehicle and its support structure are considered. But a calibration process with the mounted UCA in the use case is

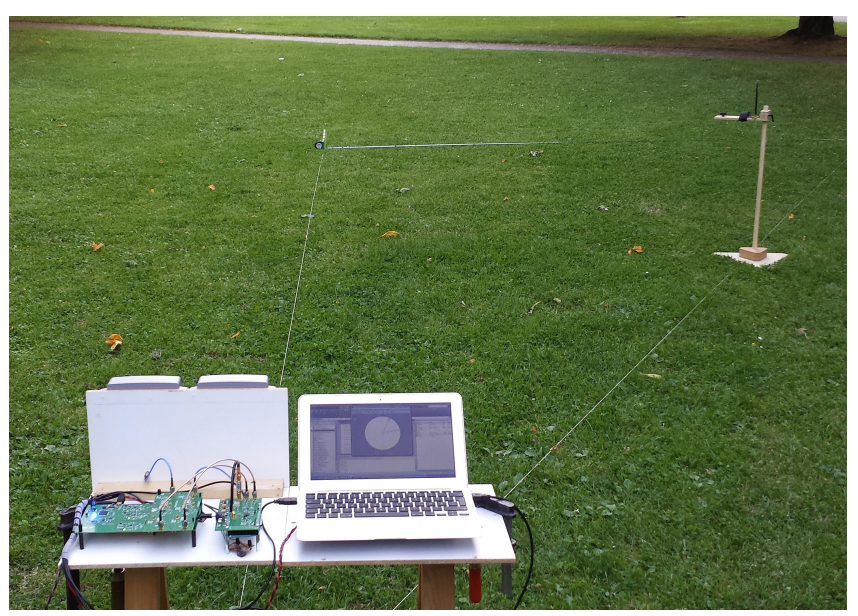

Figure 7. Measurement setup with handheld-receiver prototype.

an improvement of $19 \%$, compared to using an ideal manifold. A tolerance of $15^{\circ}$ might be huge, but it is for an initial walking direction in an open outdoor environment sufficient. In addition, these are results from a static scenario without movement of the mowing machine and could be improved just by several observations in a dynamic scenario, e.g. with tracking.

\subsection{Hand-held receiver}

The hand-held receiver can be used to guide a person to a needed distance of $0.5 \mathrm{~m}$ only by DOA estimation. Equation (6) shows that with smaller target distances the phase deviation compared to plane waves is increasing. With a distance of $0.5 \mathrm{~m}$ the phase deviation is $\delta_{\mathrm{tol}} \geq 23.4^{\circ}$, which implicates higher DOA errors. On this account, measurements are made without influences of the ground or additional elevation.

The hand-held receiver was positioned in an outdoor environment in $80 \mathrm{~cm}$ height. The transponder with a vertical oriented dipole antenna was also arranged in $80 \mathrm{~cm}$ height. Between -30 and $30^{\circ}$ in heading direction with $10^{\circ}$-steps and a distance $d$ of $10,5,2,1$ and $0.5 \mathrm{~m}$, several measurements have been made, as shown in Fig. 7. In every of these 35 different positions, 5 measurements are made without rearranging the transmit antenna.

Within a tolerance of $10^{\circ}$, every measurement point was estimated correctly. $8.9^{\circ}$ was the maximum direction error. The standard deviation of direction errors within a certain direction was $1.17^{\circ}$ minimum and $2.40^{\circ}$ maximum, as it is shown in Table 3. There are mean direction errors of minimum $0.45^{\circ}$ in direction $-20^{\circ}$ and maximum $4.77^{\circ}$ in direction $0^{\circ}$. As a function of the distance, the standard deviation of estimated direction goes from 2.2 to $3.61^{\circ}$. With a distance of $0.5 \mathrm{~m}$, the standard deviation was only $3.61^{\circ}$, as it shows Table 4. 
Table 3. Mean and standard deviation of estimated directions, direction-dependent, handheld-receiver.

\begin{tabular}{lrr}
\hline$\theta\left[^{\circ}\right]$ & $\mathbf{E}\{\hat{\theta}\}\left[^{\circ}\right]$ & $\sigma\left[^{\circ}\right]$ \\
\hline-30 & -28.72 & 2.09 \\
-20 & -20.45 & 2.30 \\
-10 & -12.3 & 1.23 \\
0 & -4.77 & 1.17 \\
10.0 & 6.08 & 1.90 \\
20.0 & 17.04 & 2.40 \\
30.0 & 25.01 & 2.35 \\
\hline
\end{tabular}

Table 4. Mean error and standard deviation of estimated directions, distance-dependent, handheld-receiver.

\begin{tabular}{lrrrrr}
\hline$d[\mathrm{~m}]$ & 0.5 & 1 & 2 & 5 & 10 \\
\hline $\mathbf{E}\{|\hat{\theta}-\theta|\}\left[^{\circ}\right]$ & 3.54 & 3.74 & 3.66 & 2.79 & 2.75 \\
$\sigma\left[^{\circ}\right]$ & 3.61 & 2.49 & 3.12 & 2.20 & 2.75 \\
\hline
\end{tabular}

The total mean direction error of all 35 measurement positions is $-2.58^{\circ}$ and standard deviation is $2.89^{\circ}$. These are the results without calibration process for the patch antenna array. Only the receiver was calibrated after 10 measurements for correct phase measurement. With a tolerance of $10^{\circ}$, a user would be able to find a fawn with the hand-held receiver, so further considerations are meaningful.

\section{Conclusion and outlook}

In this paper we presented an active RFID localization system, which enables a mowing machine driver to detect and localize RFID-marked fawns. The active RFID-system works in the UHF band at $868 \mathrm{MHz}$. A mowing machine mounted direction-of-arrival system was realized with an eight-channel-receiver and a seven-element uniform circular array to estimate the direction-of-arrival of the transponder signals in every azimuthal direction. The additional handheld receiver with a two-channel-receiver and a two-element linear patch array shall enable the user to find marked fawns by a guided approach close to a distance of $0.5 \mathrm{~m}$.

The communication concept, as shown in Sect. 3, is a simple solution for an active RFID system, in which transponder and reader are out of range the most of the lifetime. In dependency of sleep interval time the trade-off between the maximum lifetime and the maximum duration for transponder acquisition can be adjusted. A sequential eradication of many transponders in range avoids the need of a high angular resolution.

The mowing machine mounted device was tested under real world conditions and with the antenna array mounted only with a distance of $4 \mathrm{~cm}$ to the vehicle's roof. It could be shown that a calibration procedure including the mowing ma- chine leads to a big performance improvement and within a direction tolerance of $15^{\circ}, 83.44 \%$ of all measurement points could be estimated correctly. Furthermore, all these results were independent of all tested DOA algorithms.

The hand-held device has been tested under simple conditions and showed a very good performance. Within a $10^{\circ}$ direction tolerance, every measurement point could be estimated correctly. In a short distance of $0.5 \mathrm{~m}$, the mean error was $3.54^{\circ}$ without calibration procedure.

Future work will be a more detailed investigation on the possibilities for mounting a uniform circular array close to a vehicle's roof. One possibility could be determining the mutual coupling factors between antenna elements by modern calibration algorithms and separating these influences from the distortions caused by the support structure. For the handheld device real world conditions will be tested.

Acknowledgements. The project is supported by funds of the Federal Ministry of Food, Agriculture and Consumer Protection (BMELV) based on a decision of the Parliament of the Federal Republic of Germany via the Federal Office for Agriculture and Food (BLE) under the innovation support programme. We would like to thank Roland Kröner and Stefan Edstaller for many hardware realizations.

Edited by: J. Anders

Reviewed by: three anonymous referees

\section{References}

Fackelmeier, A. and Biebl, E.: A multistatic radar array for detecting wild animals during pasture mowing, in: European Radar Conference, Rome, Italy, 30 September-2 October 2009, 477 480, 2009.

Finkenzeller, K.: RFID-Handbuch - Grundlagen und praktische Anwendungen von Transpondern, kontaktlosen Chipkarten und NFC, Hanser, 5. aktualisierte und erweiterte Auflage, Carl Hanser Verlag, München, Germany, 2008.

Israel, M.: A Uav-Based Roe Deer Fawn Detection System, Proceedings of the International Conference on Unmanned Aerical Vehicle in Geomatics (UAV-g), Zürich, Switzerland, 14-16 September 2001, XXXVIII-1/C22, 1-5, doi:10.5194/isprsarchives-XXXVIII-1-C22-51-2011, 2011.

Jarnemo, A.: Roe deer Capreolus carpreolus fawns and mowing mortality rates and countermeasurements, Wildlife Biol., 8, 211 218, 2002.

Pierre, J. and Kaveh, M.: Experimental evaluation of highresolution direction-finding algorithms using a calibrated sensor array testbed, Digit. Signal Process., 5, 243-254, 1995.

Reichthalhammer, T.: Ein Radar mit synthetischer Apertur für den Nahbereich, Verlag Dr. Hut, München, Germany, 2012.

Rieck, W.: Die Setzzeit bei Reh-, Rot- und Damwild in Mitteleuropa, Zeitschrift für Jagdwissenschaft, 1, 69-75, 1955.

Schmidt, R. O.: Multiple emitter location and signal parameter estimation, Antennas and Propagation, IEEE Transactions, 34, 276 280, 1986. 
Tank, V., Haschberger, P., Dietl, H., and Lutz, W.: Infrarotoptischer Wildsensor - eine Entwicklung zur Detektion von Wild in Wiesen und zur Wildrettung bei der Frühjahrsmahd, Zeitschrift für Jagdwissenschaft, 38, 252-261, 1992.

Tuncer, E. and Friedlander, B.: Classical and Modern Direction-ofArrival Estimation, Academic Press, New York, USA, 2009.
Zekavat, R. and Buehrer, R. M.: Handbook of position location: theory, practice and advances, John Wiley \& Sons, Hoboken, New Jersey, USA, 2011. 